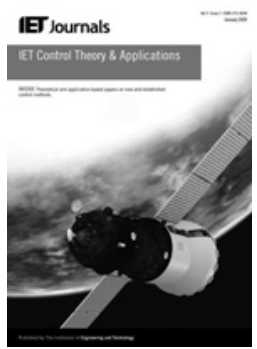

ISSN 1751-8644

\title{
Charge feedback-based robust position tracking control for piezoelectric actuators
}

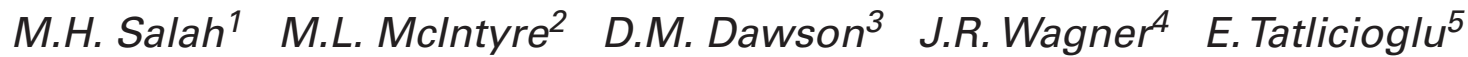 \\ ${ }^{1}$ Department of Mechatronics Engineering, Hashemite University, Zarqa, Jordan \\ ${ }^{2}$ Department of Engineering, Western Kentucky University, Bowling Green, KY, USA \\ ${ }^{3}$ Department of Electrical and Computer Engineering, Clemson University, Clemson, SC, USA \\ ${ }^{4}$ Department of Mechanical Engineering, Clemson University, Clemson, SC, USA \\ ${ }^{5}$ Department of Electrical and Electronics Engineering, Izmir Institute of Technology, Izmir, Turkey \\ E-mail: msalah@hu.edu.jo
}

\begin{abstract}
In this study, the Coleman-hysteresis model is utilised in the piezoelectric actuator (PZTA) dynamic model and a non-linear robust control strategy is then developed to actively control the displacement of the PZTA effective tip. The proposed control technique is designed based on the partial knowledge of the hysteresis model while the mass of the PZTA is assumed to be uncertain. The piezoelectric charge measurement is utilised in the controller design to reduce the effects of the hysteresis. Lyapunov-based stability analysis techniques are utilised to ensure that a desired displacement trajectory is accurately tracked. Representative numerical results are presented and discussed to demonstrate the tracking performance of several desired displacement trajectories with different frequencies and amplitudes. Finally, comparisons with a standard PID controller and a sliding mode controller were performed to examine the effectiveness of the proposed control design.
\end{abstract}

\section{Introduction}

Piezoelectric actuator (PZTA)-based systems are emerging as an important technology for precise positioning and have received wide attention in both the scientific and industrial communities. The main advantages of the PZTAs are: (i) no wear, (ii) high efficiency, (iii) almost infinite small positioning ability, (iv) ultra-fast expansion and (v) capability to deliver large actuation forces [1]. PZTAs are capable of completing high-precision actuation tasks and they are often utilised in motion actuation applications, because of their high stiffness, fast response and physically unlimited resolution [2] and can be used as either sensors or actuators in control systems [3]. They play an important role in many vital applications such as scanning tunnelling microscopy [4], scanning probe microscopy [5, 6], laser applications [7], hydraulic servo control systems [8] and hard disk drives [9-15].

The main challenging issue in precision positioning with the PZTAs is the strong hysterical activity because of their composition from ferroelectric ceramic materials [16]. Specifically, an applied voltage is typically the input control signal which activates the PZTA. In the event that the input control voltage is relatively large, the PZTA exhibits a significant amount of distortion because of the inherent hysteresis in the device, and this effect may reduce the stability of the system in feedback control applications [17]. Owing to this non-linear behaviour, the accuracy of the PZTAs are deteriorated in precise tracking control applications [16]. Hence, efficient non-linear control strategies are needed to ensure stability and to guarantee accurate tracking for the use of the PZTAs in micro/nanopositioning systems and tracking applications.

Some of the earlier research has focused on establishing accurate PZTA dynamic models whereas other research has focused on the development of control strategies for use in precision positioning and tracking applications. In Huang and Lin [18], provided a concise literature summary with regard to modelling the PZTA's dynamics and Devasia et al. [19] provided a survey of control issues in nanopositioning including control of PZTAs. Recently, the literature has focused on the development of alternate models to describe the hysteresis within the PZTA, owing to the challenging non-linear nature of this phenomenon. In Bashash and Jalili [20], proposed a dynamic model by combining the PrandtlIshlinskii hysteresis operator with a second-order linear dynamics. In Yeh et al. [21], included a non-linear spring element into the hysteresis model and utilised a Maxwellslip structure, whereas Ji et al. [22] used a support vector regression non-linear model and neural networks. In Lining et al. [23], proposed a new mathematical model to describe complex hysteresis that is based on a new parameter called the turning voltage of a PZTA. They were able to utilise this parameter to suppress the inherent hysteresis to within $\pm 1 \%$ full span range of a PZTA. In Shieh et al. [24], developed a parametrised hysteretic friction function, based on the LuGre model, to describe the PZTA hysteresis behaviour, and more recently, $\mathrm{Ru}$ et al. [25] proposed a new mathematical model for the hysteresis by using polynomial fitting. Owing to the dependence of the models in [23-25] on constant 
parameters, adaptive displacement tracking controllers with parameter adaptation algorithms were designed.

Some of the earlier research [17, 26-29] was devoted to improve the PZTA control strategies by reducing the effects of the non-linearities in the PZTA dynamic models which was achieved by applying electric charge feedback information instead of applied voltage. In [26, 27], researchers demonstrated that voltage to displacement linearisation of a PZTA may be achieved if the control input is the applied electric charge rather than the applied voltage. Furutani et al. [28] was able to improve the PZTA control strategy by combining induced charge feedback with an inverse transfer function compensation. Vautier and Moheimani [17] showed that applying an electric charge to control the position reduces the effects of the non-linearity in the PZTA dynamics. Further, in [29], Main et al. presented experimental data from tests for both voltage and charge control which showed that charge control is significantly more linear and less hysteretic than voltage control over the same actuator displacement range.

Other research in the literature has focused on the development of intelligent control schemes for the precision control of PZTAs [1, 2, 30-37]. Neural networks and fuzzy controls were also utilised to model the PZTA hysteresis non-linearities and control the micro/nano motion of the PZTA [1, 32]. Liaw et al. [2] proposed a robust control strategy for precise positioning tracking. The implementation of their control law required only a knowledge of the estimated system parameters and their corresponding bounds as well as the bound of the hysteresis effect including disturbances. Stepanenko and $\mathrm{Su}$ [31] introduced and implemented an approximation function to compensate for the hysteresis non-linearities by fuzzy logic techniques. Some of these designs were based on the inverse hysteresis model that is assumed to be known a priori, so feedforward techniques can be utilised in the control design [30, 33]. In [1, 34-36], feedback linearisation was utilised to compensate for the hysteresis dynamics and then tracking controllers were implemented. Wu and Zou [37] presented an inversion-based iterative control approach to compensate for both the hysteresis and the vibrational dynamics variations during high-speed, large-range tracking. The focus of recent research was devoted to the design and application of robust control techniques to PZTA systems for micro/nanomanipulation [20, 38-44].

In this paper, the displacement of a PZTA is actively controlled to track a desired trajectory. The dynamic model of the PZTA is based on Coleman-Hodgdon hysteresis model in [45]. Since charge feedback controllers reduce the effects of the non-linearities in the PZTA dynamic model and provide better performance over the voltage feedback controllers [17, 26-29, 46] the electric charge feedback is utilised in the controller design to reduce the effects of the hysteresis in the PZTA dynamic model. The feedback charge of the PZTA is obtained by measuring the voltage across a capacitor that is added in-series to the PZTA circuit. A non-linear robust control strategy is then developed based on the partial knowledge of the hysteresis model. In the control design, the mass of the PZTA is assumed to be uncertain. Lyapunov-based tools are utilised in the development of the robust controller and to prove tracking of a desired trajectory by practically regulating the error signal to zero. Representative numerical results are presented for different desired trajectories to demonstrate the proof of concept of the active control approach. This rest of the paper is organised as follows. In Section 2, a Coleman-Hodgdon-based hysteresis model along with the PZTA dynamic model are presented. In Section 3, a non-linear robust control scheme is developed along with the stability analysis which verifies that the piezoelectric desired displacement can be tracked. In Section 4, numerical results are presented to demonstrate the performance of the proposed robust control strategy and the concluding remarks are provided in Section 5.

\section{PZTA system model}

\subsection{PZTA elongation dynamics}

A PZTA with a single elongation axis, depicted in Fig. 1, can be dynamically modelled as

$$
m \ddot{y}+F_{L}=F_{p}
$$

where $m \in \mathbb{R}$ is the PZTA mass, $L \in \mathbb{R}$ is the nonactivated length of the PZTA, $F_{p}(t) \in \mathbb{R}$ is the force generated by the PZTA elongation, $F_{L}(y, \dot{y}) \in \mathbb{R}$ is the perpendicular forces acting on the PZTA, $y(t), \dot{y}(t), \ddot{y}(t) \in \mathbb{R}$ are the displacement, velocity and acceleration of the PZTA effective tip of elongation, respectively. An equivalent circuit model of the PZTA can be presented as shown in Fig. 2.

In the equivalent model in Fig. $2, V(t) \in \mathbb{R}$ is the applied input control voltage, $\dot{q}(t)=i(t) \in \mathbb{R}$ represents the current flowing through the PZTA, $C_{\mathrm{m}} \in \mathbb{R}^{+}$is a series connected capacitor that facilitates the measurement of the induced charge $q(t) \in \mathbb{R}$, through a measurement of the voltage $V_{\mathrm{m}}(t) \in \mathbb{R}$ by utilising $q=C_{\mathrm{m}} V_{\mathrm{m}}$. In Fig. 2, the circuit element indicated by $H$ models the inherent hysteresis between the voltage $V_{\mathrm{h}}(t) \in \mathbb{R}$ across it and the induced charge $q(t)$. The parameter $C_{\mathrm{c}} \in \mathbb{R}^{+}$is the internal capacitance of the PZTA, $\dot{q}_{\mathrm{c}}(t) \in \mathbb{R}$ is the current flowing through $C_{\mathrm{c}}$ and $V_{\mathrm{c}}(t) \in \mathbb{R}$ is the voltage across it. The circuit element indicated by $T_{\mathrm{em}}$ represents the elongation model, where $T_{\mathrm{em}} \in \mathbb{R}^{+}$is the elongation constant inherent to the PZTA [2], and $\dot{q}_{p}(t) \in \mathbb{R}$ denotes the current flowing through this circuit branch.

\subsection{Hysteresis model}

A non-linear hysteresis model $H\left(V_{\mathrm{h}}\right) \in \mathbb{R}$ can be defined to describe the relationship between the input voltage $V_{\mathrm{h}}(t)$ and the induced charge $q(t)$. The following hysteresis model will

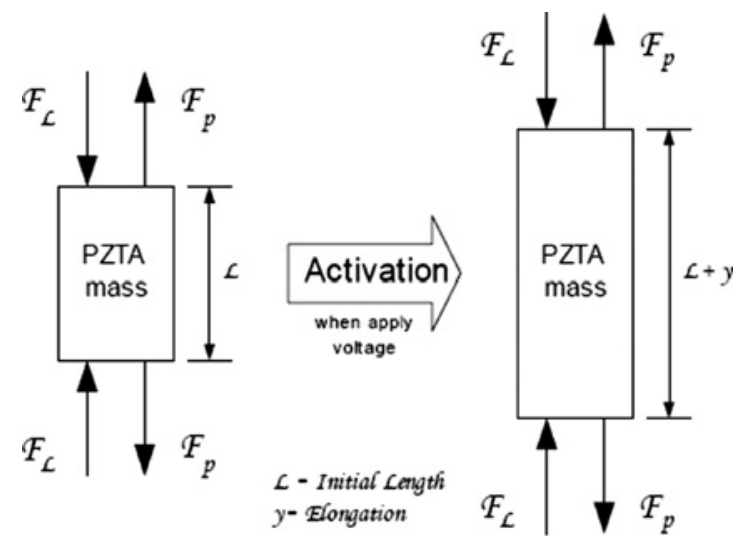

Fig. 1 PZTA elongation induced by applied voltage 


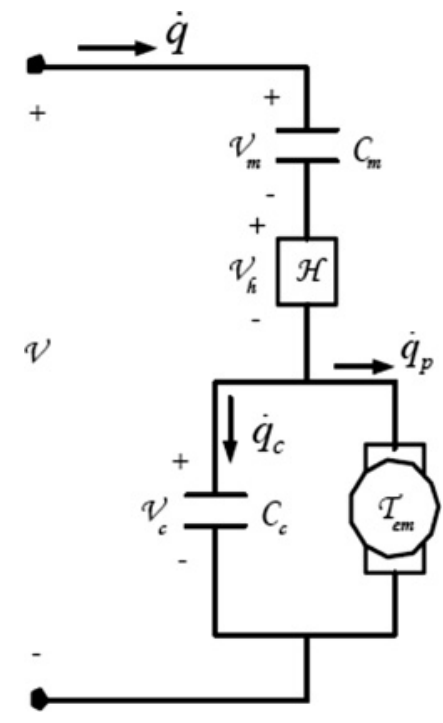

Fig. 2 PZTA equivalent circuit model

be utilised $[45,47]$

$$
q=H\left(V_{\mathrm{h}}\right) \triangleq f\left(V_{\mathrm{h}}\right)+d\left(V_{\mathrm{h}}\right)
$$

where $f\left(V_{\mathrm{h}}\right) \in \mathbb{R}$ is a subsequently defined signal [see e.g. (36)]. When $V_{\mathrm{h}}(t)$ is increasing (i.e. when $\dot{V}_{\mathrm{h}}(t)>0$ and $\left.V_{\mathrm{h}}(t)>V_{\mathrm{h}_{o}}\right)$ the function $d\left(V_{\mathrm{h}}\right) \in \mathbb{R}$ is defined as follows

$$
\begin{aligned}
d(\cdot) \triangleq & {\left[q_{o}-f\left(V_{\mathrm{h}_{o}}\right)\right] e^{-\delta\left(V_{\mathrm{h}}-V_{\mathrm{h}_{o}}\right)} } \\
& +e^{-\delta V_{\mathrm{h}}} \int_{V_{\mathrm{h}_{o}}}^{V_{\mathrm{h}}}\left(g(\tau)-f^{\prime}(\tau)\right) e^{\delta \tau} \mathrm{d} \tau
\end{aligned}
$$

and when $V_{\mathrm{h}}(t)$ is decreasing (i.e. when $\dot{V}_{\mathrm{h}}(t)<0$ and $\left.V_{\mathrm{h}}(t)<V_{\mathrm{h}_{o}}\right)$ the function $d\left(V_{\mathrm{h}}\right) \in \mathbb{R}$ is defined as follows

$$
\begin{aligned}
d(\cdot) \triangleq & {\left[q_{o}-f\left(V_{\mathrm{h}_{o}}\right)\right] e^{-\delta\left(V_{\mathrm{h}_{o}}-V_{\mathrm{h}}\right)} } \\
& +e^{\delta V_{\mathrm{h}}} \int_{V_{\mathrm{h}_{o}}}^{V_{\mathrm{h}}}\left(f^{\prime}(\tau)-g(\tau)\right) e^{-\delta \tau} \mathrm{d} \tau
\end{aligned}
$$

where $q_{o} \in \mathbb{R}$ is the induced charge at $t=t_{o}, V_{\mathrm{h}_{o}} \in \mathbb{R}$ is the input voltage at $t=t_{o}, \delta \in \mathbb{R}^{+}$is a constant, $f^{\prime}(\cdot)=$ $\partial f\left(V_{\mathrm{h}}\right) / \partial V_{\mathrm{h}}$ and $g(\cdot) \in \mathbb{R}$ a subsequently designed signal [see e.g. (37)]. The signals $d\left(V_{\mathrm{h}}\right), f\left(V_{\mathrm{h}}\right)$ and $g\left(V_{\mathrm{h}}\right) \in \mathbb{R}$ have the following properties [47]:

Property 1: The function $f\left(V_{\mathrm{h}}\right)$ is piecewise smooth, monotone increasing and odd.

Property 2: The function $g\left(V_{\mathrm{h}}\right)$ is piecewise continuous, and even.

Property 3: The function $f\left(V_{\mathrm{h}}\right)$, is known, and invertible, such that $\bar{V}_{\mathrm{h}}=f\left(V_{\mathrm{h}}\right)$, and $V_{\mathrm{h}}=f^{-1}\left(\bar{V}_{\mathrm{h}}\right)$.

Property 4: The function $f^{\prime}(\cdot)$ is not identically zero, hence, $f^{\prime}(\infty)=\eta_{o}$, where $\eta_{o} \in \mathbb{R}^{+}$is a constant.

Property 5: The function $g\left(V_{\mathrm{h}}\right)$ has a finite upper limit [i.e. $f^{\prime}(\infty)=g(\infty)$ ] where $f^{\prime}\left(V_{\mathrm{h}}\right) \geq g\left(V_{\mathrm{h}}\right)$.

Property 6: The function $d\left(V_{\mathrm{h}}\right)$ is bounded as

$$
|d| \leq \eta_{\mathrm{d}}
$$

where $\eta_{\mathrm{d}} \in \mathbb{R}^{+}$is a constant (see Appendix 1 for proof).

\subsection{Elongation model}

The elongation model of the PZTA is described by the following two linear relationships [2]. First relationship models the effect of the induced charge, denoted by $q_{p}(t)$, on the displacement of the elongation axis and is described as follows

$$
q_{p} \triangleq T_{\mathrm{em}} y
$$

and the second relationship depicts the force $F_{p}(t)$ imparted by the elongation action as a function of the voltage $V_{\mathrm{c}}(t)$ as follows [2]

$$
F_{p} \triangleq T_{\mathrm{em}} V_{\mathrm{c}}
$$

\subsection{Dynamic model}

To facilitate the subsequent control objective, a dynamic expression is aimed that relates the displacement of the elongation axis $y(t)$ as a function of charge $q(t)$ induced within the PZTA. The advantage of working with such a dynamic model is clear owing to the lack of the hysteresis terms, which has been discussed in the literature [17, 28, 29]. From Fig. 2, it is clear that the induced charge $q(t)$ can be described as

$$
q=C_{\mathrm{c}} V_{\mathrm{c}}+q_{p}
$$

After utilising (8), the elongation dynamics in (1) can be written as

$$
m \ddot{y}+\bar{F}_{L}=\left(\frac{T_{\mathrm{em}}}{C_{\mathrm{c}}}\right) q
$$

where (6) and (7) were utilised and the variable $\bar{F}_{L}(y, \dot{y}) \in \mathbb{R}$ is defined as

$$
\bar{F}_{L} \triangleq F_{L}+\left(\frac{T_{\mathrm{em}}^{2}}{C_{\mathrm{c}}}\right) y
$$

To facilitate the tracking control design, three assumptions frame the analysis.

Assumption 1: The parameters $C_{\mathrm{c}}, C_{\mathrm{m}}$ and $T_{\mathrm{em}}$ are assumed to be known, and constants with respect to time.

Assumption 2: The velocity $\dot{y}(t)$ and displacement $y(t)$ of the PZTA effective tip are assumed to be measurable. The velocity measurement can be manufactured digitally using $y(t)$ and the backwards differentiation algorithm, or a variable structure observer can be used [48].

Assumption 3: It is assumed that the forces $F_{L}(y, \dot{y})$ and their first time derivative $\dot{F}_{L}(\cdot)$ are bounded provided that $y(t)$, $\dot{y}(t)$ and $\ddot{y}(t)$ are bounded.

\section{Robust control development}

\subsection{Control design objective}

The control objective is to ensure that the displacement $y(t)$ of the PZTA effective tip tracks the desired trajectory $y_{\mathrm{d}}(t) \in$ $\mathbb{R}$ in the following sense

$$
\left|y_{\mathrm{d}}(t)-y(t)\right| \leq \varepsilon \text { as } t \rightarrow \infty
$$

where $\varepsilon \in \mathbb{R}^{+}$is a constant that can be selected arbitrary small and also guaranteeing that all the system signals remain bounded under the closed-loop operation. 


\section{www.ietdl.org}

Assumption 4: The subsequent analysis requires the standard assumption that the desired trajectory is selected such that $y_{\mathrm{d}}(t), \dot{y}_{\mathrm{d}}(t), \ddot{y}_{\mathrm{d}}(t)$ are bounded.

To facilitate the subsequent development, a filtered tracking error signal, denoted by $r(t) \in \mathbb{R}$, is defined as

$$
r \triangleq \dot{e}+\alpha e
$$

where $\alpha \in \mathbb{R}^{+}$is a control gain and the displacement tracking error, denoted by $e(t) \in \mathbb{R}$, is defined as

$$
e \triangleq y_{\mathrm{d}}-y
$$

Based on the definition of $e(t)$ in (13), it is clear that if $|e(t)| \leq \varepsilon$, then $\left|y_{\mathrm{d}}(t)-y(t)\right| \leq \varepsilon$, thus, meeting the control objective.

\subsection{Closed-loop error system}

To facilitate the development of a closed-loop error system, a control strategy must be developed to account for the inherent hysteresis which exists between the input voltage $V_{\mathrm{h}}(t)$ and the induced charge $q(t)$. It is clear from Fig. 2 that an expression for $V_{\mathrm{h}}(t)$ can be obtained as follows

$$
V_{\mathrm{h}}=V-V_{\mathrm{m}}-V_{\mathrm{c}}
$$

After utilising (14), the charge expression in (2) can be rewritten as follows

$$
q=f\left(V-V_{\mathrm{m}}-V_{\mathrm{c}}\right)+d\left(V_{\mathrm{h}}\right)
$$

To meet the tracking control objective, the control input $V(t)$ is designed as follows

$$
V \triangleq V_{\mathrm{m}}+V_{\mathrm{c}}+f^{-1}\left(\bar{V}_{\mathrm{h}}\right)
$$

where $\bar{V}_{\mathrm{h}}(t) \in \mathbb{R}$ is a subsequently designed auxiliary control signal, and Property 3 was utilised.

Remark 1: From (8), the voltage $V_{\mathrm{c}}(t)$ can be obtained as

$$
V_{\mathrm{c}}=\frac{1}{C_{\mathrm{c}}}\left(q-q_{p}\right)
$$

where the charge $q_{p}(t)$ is computed from (6), and the charge $q(t)$ is computed from the measurement of $V_{\mathrm{m}}(t)$ across the capacitor $C_{\mathrm{m}}$ (i.e. $q=C_{\mathrm{m}} V_{\mathrm{m}}$ ).

After utilising (2) and (16), the expression in (15) can be rewritten as follows

$$
q=H\left(V_{\mathrm{h}}\right) \triangleq \bar{V}_{\mathrm{h}}+d\left(V_{\mathrm{h}}\right)
$$

To facilitate the closed-loop error system development, an auxiliary error signal $s(t) \in \mathbb{R}$ is defined as

$$
s \triangleq q_{\mathrm{d}}-q
$$

where $q_{\mathrm{d}}(y, \dot{y}) \in \mathbb{R}$ is a subsequently designed desired charge. After utilising (18), the following expression can be obtained for the auxiliary error signal $s(t)$

$$
s=q_{\mathrm{d}}-\bar{V}_{\mathrm{h}}-d\left(V_{\mathrm{h}}\right)
$$

Based on (20), the auxiliary control signal, $\bar{V}_{\mathrm{h}}(t)$ is designed as

$$
\bar{V}_{\mathrm{h}} \triangleq q_{\mathrm{d}}+k_{S} s
$$

where $k_{s} \in \mathbb{R}^{+}$is a constant gain.
The auxiliary control signal in (21) can be substituted into (20) to obtain the following expression

$$
s=-k_{s} s-d\left(V_{\mathrm{h}}\right)
$$

from which the auxiliary error signal $s(t)$ can be upper bounded as

$$
s \leq \frac{|d|}{1+k_{s}} \leq \eta_{s}
$$

where $\eta_{s} \in \mathbb{R}^{+}$is a constant and Property 6 was utilised.

With the control concept in place to account for the voltage to charge hysteresis, the dynamic model as defined in (9) is now incorporated to complete the closed-loop error system development. From the PZTA dynamics in (9), the following expression can be obtained

$$
m \ddot{y}+\bar{F}_{L}+\left(\frac{T_{\mathrm{em}}}{C_{\mathrm{c}}}\right) s=\left(\frac{T_{\mathrm{em}}}{C_{\mathrm{c}}}\right) q_{\mathrm{d}}
$$

where (19) was utilised. To facilitate the development of the closed-loop error system for $r(t)$, the first time derivative of (12) is taken and then both sides are multiplied by $m$, thus, obtaining the following expression

$$
m \dot{r}=m \ddot{y}_{\mathrm{d}}+\bar{F}_{L}+\left(\frac{T_{\mathrm{em}}}{C_{\mathrm{c}}}\right) s-\left(\frac{T_{\mathrm{em}}}{C_{\mathrm{c}}}\right) q_{\mathrm{d}}+\alpha m \dot{e}
$$

where the second time derivative of (13) and (24) were utilised. To facilitate the subsequent analysis, the expression in $(25)$ is rewritten as

$$
m \dot{r}=\tilde{N}+N_{\mathrm{d}}-e+\left(\frac{T_{\mathrm{em}}}{C_{\mathrm{c}}}\right) s-\left(\frac{T_{\mathrm{em}}}{C_{\mathrm{c}}}\right) q_{\mathrm{d}}
$$

where the auxiliary signal $\tilde{N}(y, \dot{y}) \in \mathbb{R}$ is defined as

$$
\tilde{N} \triangleq N-N_{\mathrm{d}}
$$

where the auxiliary signal $N\left(y, \dot{y}, \ddot{y}_{\mathrm{d}}\right) \in \mathbb{R}$ is defined as

$$
N \triangleq m \ddot{y}_{\mathrm{d}}+\bar{F}_{L}+e+\alpha m \dot{e}
$$

and the auxiliary signal $N_{\mathrm{d}}(t) \in \mathbb{R}$ is defined as

$$
\begin{aligned}
N_{\mathrm{d}} & \left.\triangleq N\right|_{y=y_{\mathrm{d}}, \dot{y}=\dot{y}_{\mathrm{d}}} \\
& =m \ddot{y}_{\mathrm{d}}+\bar{F}_{L \mathrm{~d}}
\end{aligned}
$$

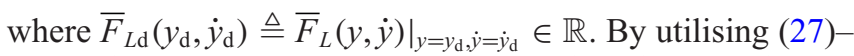
(29), the following inequality can be developed (see Appendix 3 for further details)

$$
|\tilde{N}| \leq \rho(\|z\|)\|z\|
$$

where $\rho(\|z\|) \in \mathbb{R}$ is a positive, non-decreasing function of its argument and $z(t) \in \mathbb{R}^{2}$ is the combined error signal defined as follows

$$
z=\left[\begin{array}{ll}
e & r
\end{array}\right]^{\mathrm{T}}
$$

Based on (26), the auxiliary desired charge signal $q_{\mathrm{d}}(y, \dot{y})$ is designed as

$$
q_{\mathrm{d}}=\left(\frac{C_{\mathrm{c}}}{T_{\mathrm{em}}}\right)\left[k_{r} r+\frac{1}{\varepsilon} \rho(\|z\|)^{2}\|z\|^{2} r\right]
$$

where $k_{r} \in \mathbb{R}^{+}$is a constant gain and $\varepsilon \in \mathbb{R}^{+}$is a small constant. After substituting (32) into (26), the following 
closed-loop error system can be obtained

$$
m \dot{r}=\tilde{N}+N_{\mathrm{d}}-e+\left(\frac{T_{\mathrm{em}}}{C_{\mathrm{c}}}\right) s-k_{r} r-\frac{1}{\varepsilon} \rho(\|z\|)^{2}\|z\|^{2} r
$$

It is to be noted that the controller given in (16), (21) and (32) was designed on the assumption that the mass of the PZTA is not available for control development.

\subsection{Stability analysis}

Theorem 1: The controller given in (16), (21) and (32) ensures that $|e(t)| \leq \varepsilon$ as $t \rightarrow \infty$ provided the control gain $k_{r}$, introduced in (32), is sufficiently large, and hence, $e(t)$ is practically regulated to zero. The controller also guarantees that all closed-loop signals remain bounded.

Proof: See Appendix 2.

\section{$4 \quad$ Numerical results}

Numerical simulations were performed to demonstrate the proof of concept of the proposed robust tracking controller. The PZTA dynamic model, introduced in (24), was used to implement the actuator in the numerical simulation study. The PZTA parameters were set as $C_{\mathrm{c}}=1.5 \mu \mathrm{F}$ and $T_{\mathrm{em}}=$ $1 \mathrm{~N} / \mathrm{V}$. To prove the robustness of proposed controller design, the mass of the PZTA was chosen to be a timevarying function as follows

$$
m=0.1 \sin (6000 \pi t)+0.11 \mathrm{~kg}
$$

The measurement capacitor $C_{\mathrm{m}}$ was omitted for this simulation owing to ability to measure the charge $q(t)$ within the simulation environment. The forces $F_{L}(y, \dot{y})$ on the PZTA was defined as

$$
F_{L}=k y+b \dot{y}
$$

where $k=14.2 \times 10^{6} \mathrm{~N} / \mathrm{m}$, and $b=7540 \mathrm{~N} \mathrm{~s} / \mathrm{m}$. The voltage to charge hysteresis model is implemented using the expressions in (2)-(4), where the following functions are utilised [45]

$$
\begin{aligned}
& f=A \tanh \left(\xi V_{\mathrm{h}}\right)+\mu_{o} V_{\mathrm{h}} \\
& g=\frac{\partial f}{\partial V_{\mathrm{h}}}\left(1-\gamma e^{-\beta\left|V_{\mathrm{h}}\right|}\right)
\end{aligned}
$$

where $\delta=0.1, A=5000, \xi=0.6, \mu_{o}=0.1, \gamma=0.6, \beta=$ 0.1 and $\tanh (\cdot)$ is the standard hyperbolic tangent function. The expressions selected for $f\left(V_{\mathrm{h}}\right)$ and $g\left(V_{\mathrm{h}}\right)$ in (36) and (37) satisfy Properties 1-5.

The proposed controller was first tested in tracking a variable amplitude desired trajectory as shown in Fig. 3. The control gains were tuned and chosen as $\alpha=2 \times 10^{5}$, $k_{r}=10^{5}, k_{s}=100, \varepsilon=10^{-5}, \rho=100$ and the sampling frequency was chosen as $10^{-6} \mathrm{~Hz}$. The PZTA desired trajectory $y_{\mathrm{d}}(t)$ against actual displacement $y(t)$, tracking error $e(t)$, voltage $V(t)$ and the hysteresis response are presented in Figs. 3-6, respectively. These numerical simulation results clearly indicate that the proposed robust controller can track variable amplitude desired trajectory where $2.5 \%$ maximum transient tracking error and $0.8 \%$ maximum steady-state error were achieved.

\subsection{Comparison with a PID controller}

In this section, the proposed robust controller was compared with a PID controller. The following desired trajectory was selected

$$
y_{\mathrm{d}}=3 \sin \left(2 \pi f_{\mathrm{d}} t-0.5 \pi\right)+3 \mu \mathrm{m}
$$

where $f_{\mathrm{d}} \in \mathbb{R}$ is the frequency of the desired trajectory and the initial conditions were set to be as

$$
\begin{aligned}
& q\left(t_{0}\right)=0 \mathrm{C}, \quad V\left(t_{0}\right)=0 \mathrm{~V} \\
& y\left(t_{0}\right)=3 \mu \mathrm{m}, \quad \dot{y}\left(t_{0}\right)=0
\end{aligned}
$$

where $t_{0}=0 \mathrm{~s}$. Three different frequencies (i.e. 10, 100 and $1000 \mathrm{~Hz}$ ) were selected for the desired trajectory in (38) to demonstrate bandwidth of the proposed approach. For all three different frequencies, sampling frequency was chosen as $10^{-7} \mathrm{~Hz}$, and the control gains were tuned and chosen as $\alpha=10^{4}, k_{r}=5 \times 10^{6}, k_{s}=100, \varepsilon=10^{-5}, \rho=100$. To test the effectiveness of the proposed non-linear controller in (16), (21) and (32), it was compared with a standard PID controller $\left(V_{\mathrm{h}}=k_{p} e+k_{i} \int_{t_{0}}^{t} e(\tau) \mathrm{d} \tau+k_{\mathrm{d}} \dot{e}\right)$ with gains of $k_{p}=k_{i}=5 \times 10^{6}, k_{\mathrm{d}}=1 \times 10^{3}$. The tracking errors $e(t)$ are presented in Figs. 7-9, for the selected frequency $f_{\mathrm{d}}=10 \mathrm{~Hz}, f_{\mathrm{d}}=100 \mathrm{~Hz}$ and $f_{\mathrm{d}}=1000 \mathrm{~Hz}$, respectively. The control voltage inputs $V(t)$ are presented in Figs. 10-12, for the selected frequency $f_{\mathrm{d}}=10 \mathrm{~Hz}, f_{\mathrm{d}}=100 \mathrm{~Hz}$ and $f_{\mathrm{d}}=$ $1000 \mathrm{~Hz}$, respectively.

In order to quantify the performance of each controller, the following measures were computed

$$
\begin{gathered}
M_{\mathrm{e}} \triangleq \int_{t_{0}}^{t}|e(\rho)|^{2} \mathrm{~d} \rho \\
M_{\mathrm{V}} \triangleq \int_{t_{0}}^{t}|V(\rho)|^{2} \mathrm{~d} \rho
\end{gathered}
$$

where $M_{\mathrm{e}}(t)$ and $M_{\mathrm{V}}(t)$ are measures of the magnitude of the tracking error and the control voltage input, respectively, over the period of operation of the system. Table 1 shows the measures for both the proposed non-linear controller and standard PID controller. From Table 1, it is clear that the tracking performance using the robust non-linear controller was superior when compared with a standard PID controller.

\subsection{Comparison with a sliding mode controller}

In this section, the proposed robust controller was further compared with the sliding mode controller in [43]. The following multi-frequency signal was selected as the desired trajectory

$$
\begin{aligned}
y_{\mathrm{d}}= & 5.2-0.5 \cos (30 \pi t)-\cos (20 \pi t)-1.3 \cos (10 \pi t) \\
& -2.5 \cos (16 \pi t) \mu \mathrm{m}
\end{aligned}
$$

where the control gains were tuned and chosen as $\alpha=$ $2 \times 10^{5}, k_{r}=10^{5}, k_{s}=100, \varepsilon=10^{-5}, \rho=100$ and the sampling frequency was chosen as $10^{-6} \mathrm{~Hz}$. The initial condition was set $y\left(t_{0}\right)=0$ and a $10 \%$ mismatch was considered for $C_{\mathrm{c}}$ and $T_{\mathrm{em}}$. The PZTA desired trajectory $y_{\mathrm{d}}(t)$ against actual displacement $y(t)$, tracking error $e(t)$, voltage $V(t)$ and the hysteresis response are presented in 


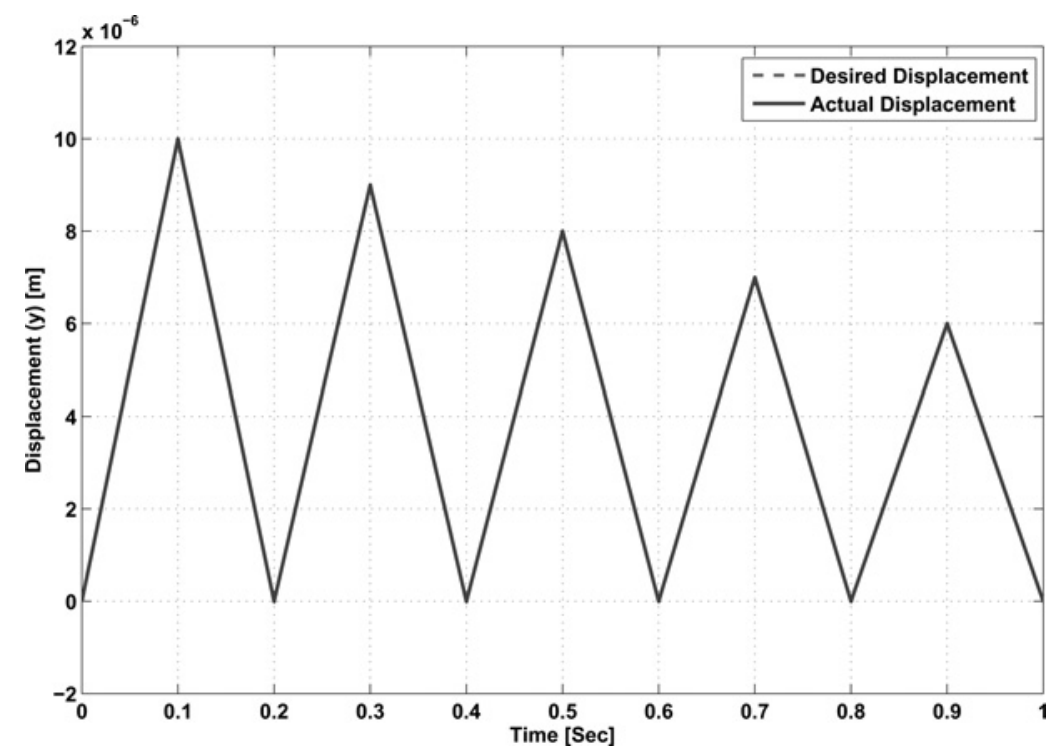

Fig. 3 PZTA variable amplitude desired trajectory $y_{d}(t)$ against actual displacement $y(t)$

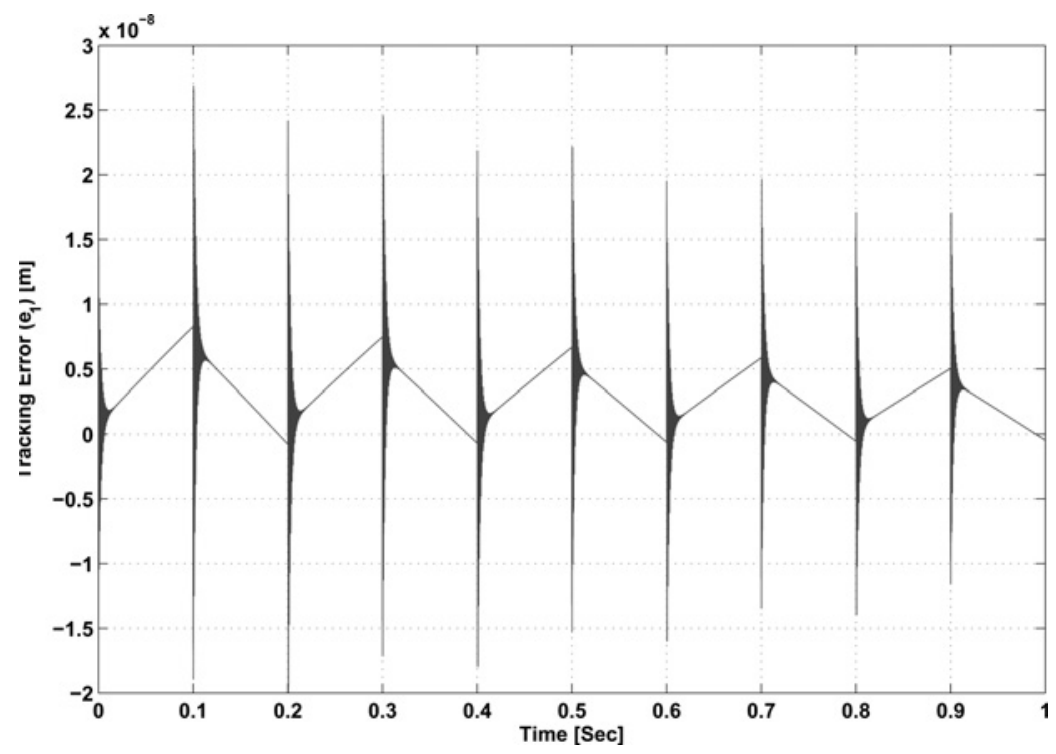

Fig. 4 PZTA tracking error e(t) for the variable amplitude desired trajectory

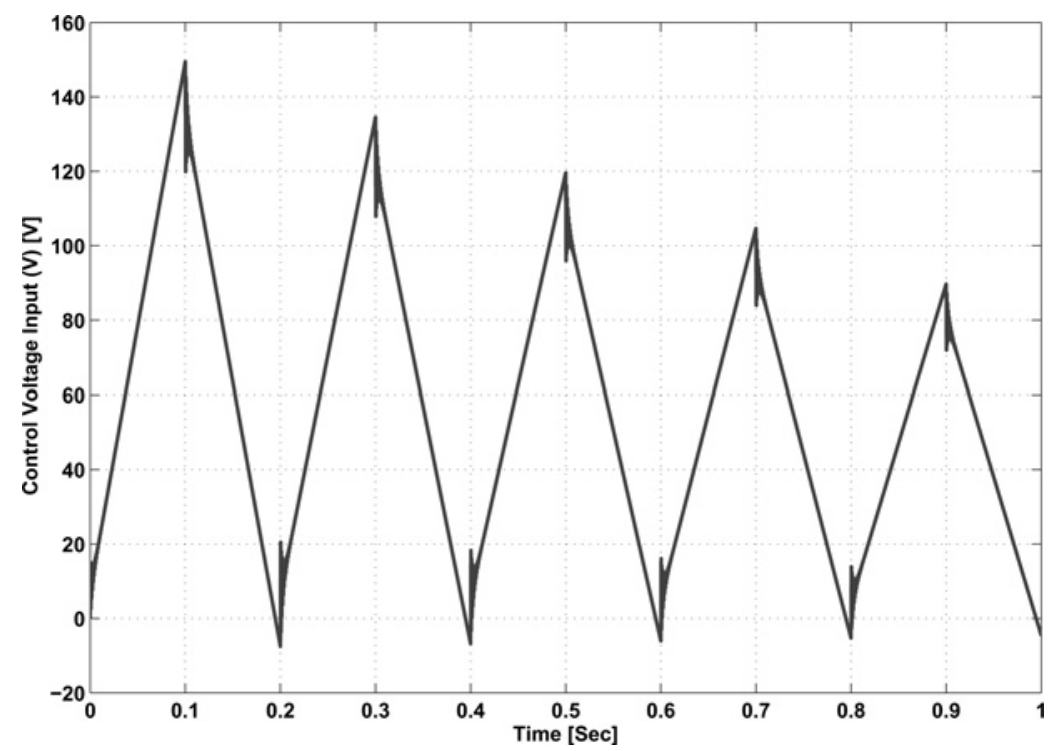

Fig. 5 Voltage $V(t)$ for the variable amplitude desired trajectory 


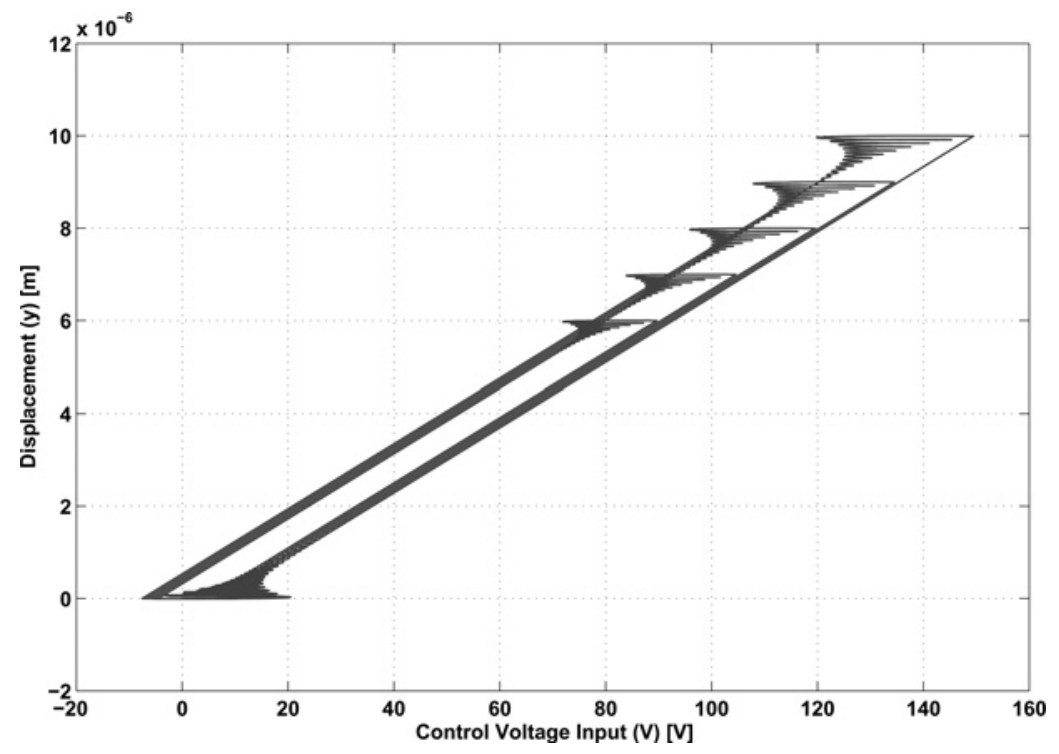

Fig. 6 Hysteresis response for the variable amplitude desired trajectory

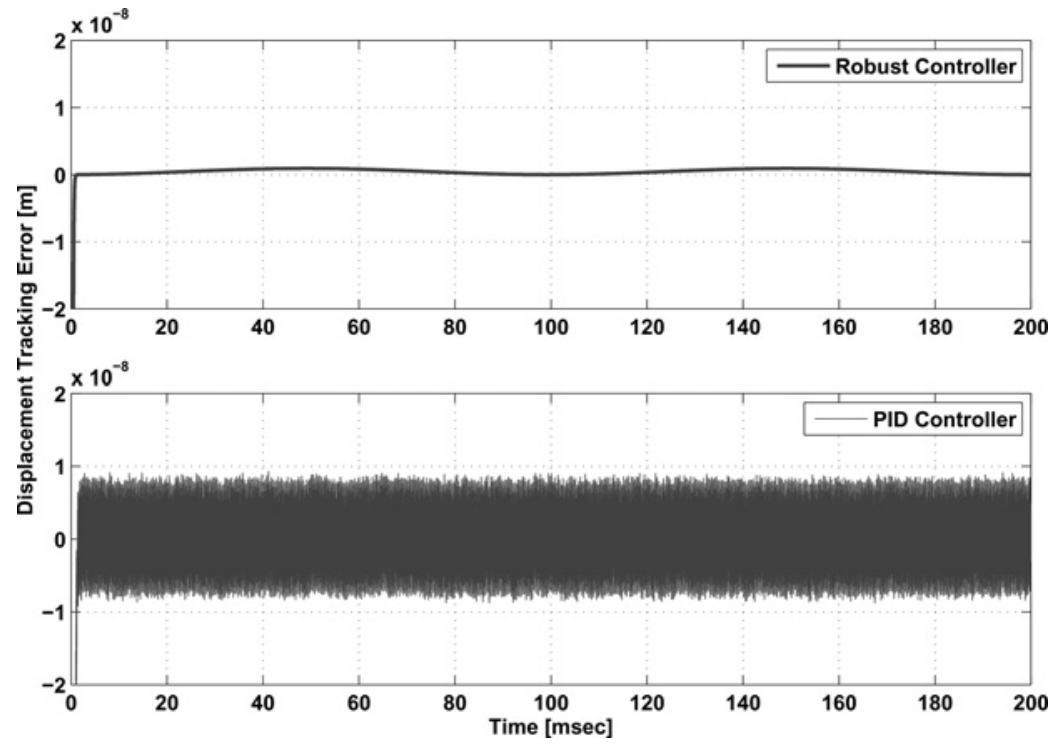

Fig. 7 PZTA tracking error e(t) for a desired frequency of $10 \mathrm{~Hz}$

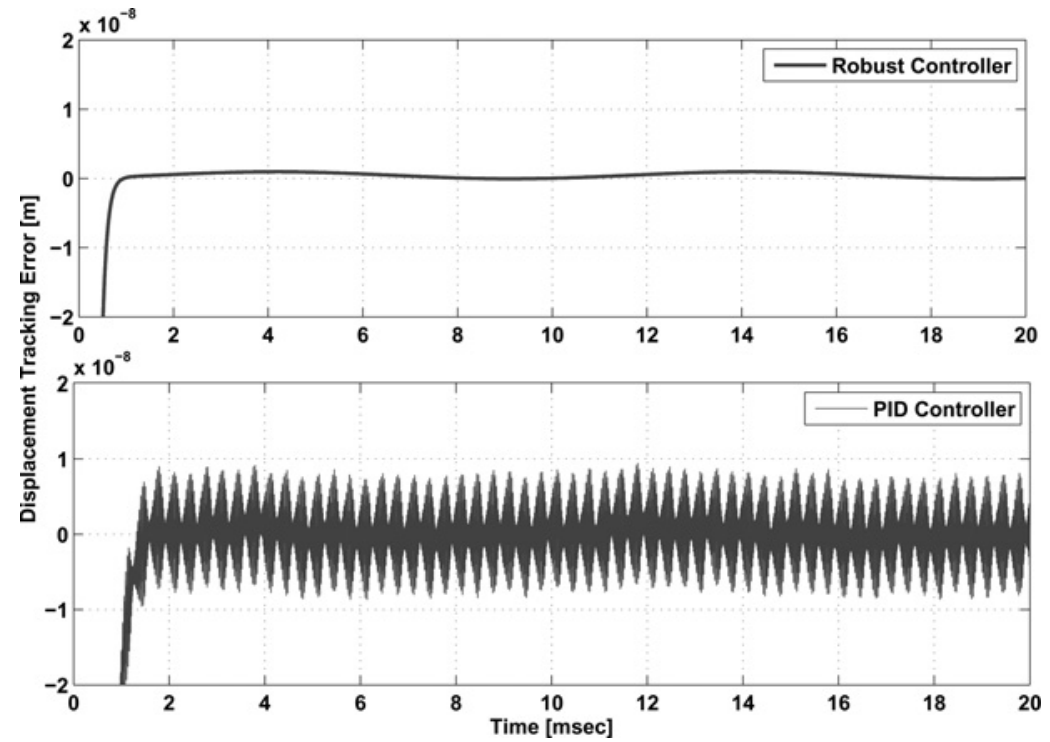

Fig. 8 PZTA tracking error e(t) for a desired frequency of $100 \mathrm{~Hz}$ 


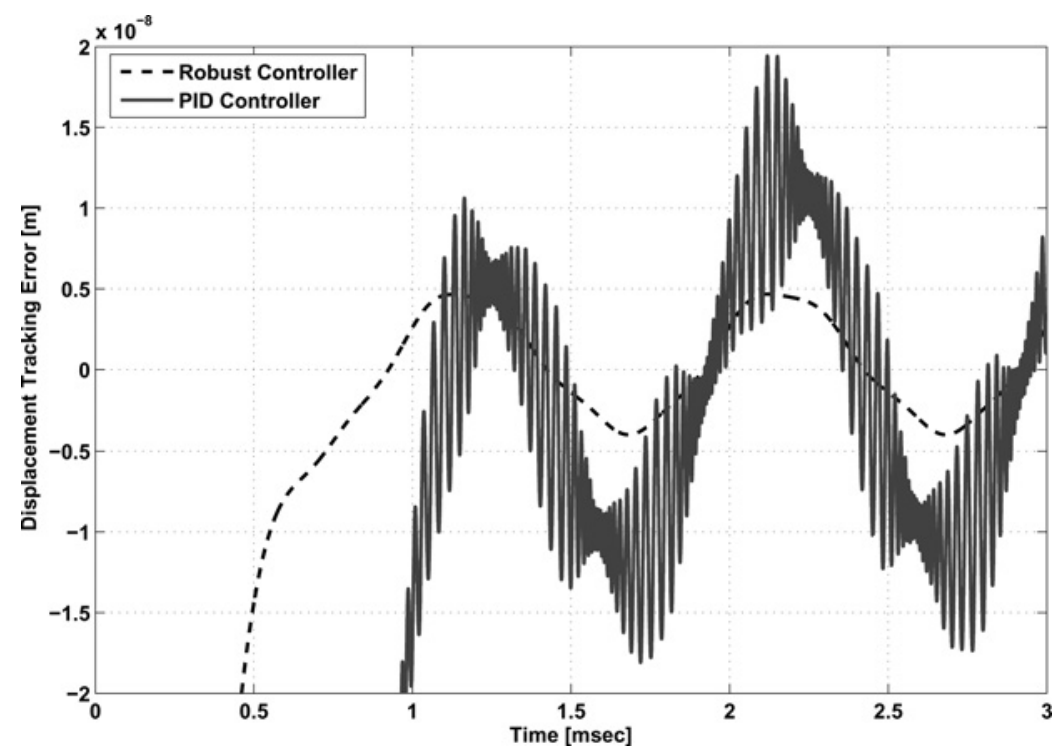

Fig.9 PZTA tracking error e(t) for a desired frequency of $1000 \mathrm{~Hz}$

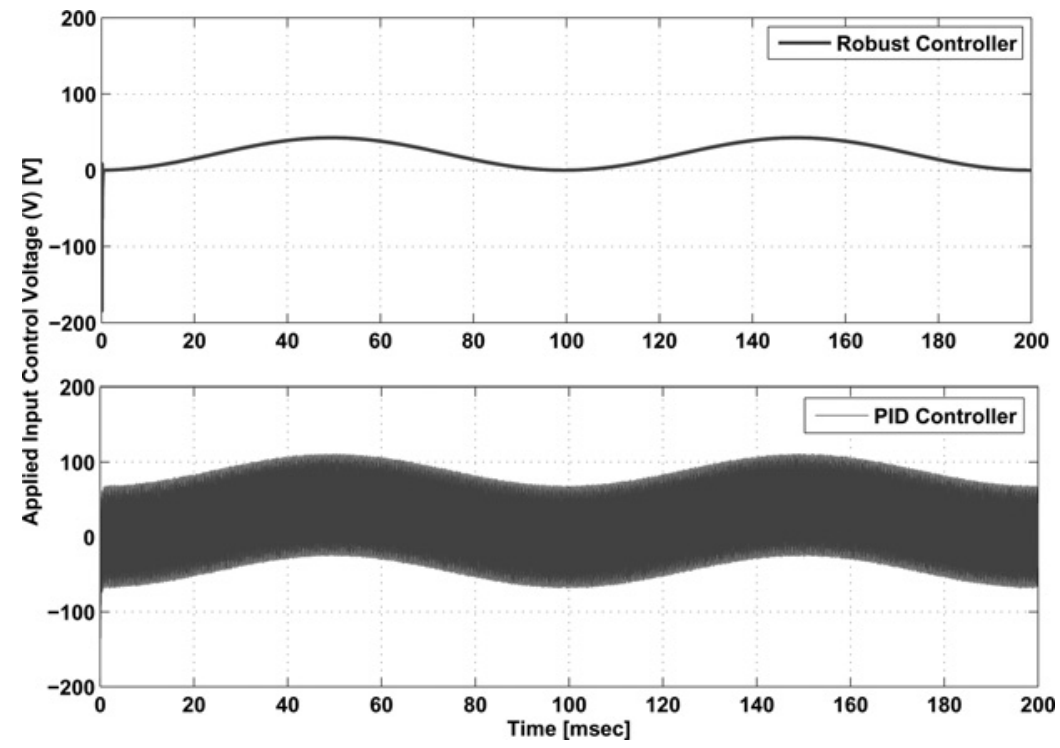

Fig. 10 Voltage $V(t)$ for a desired frequency of $10 \mathrm{~Hz}$

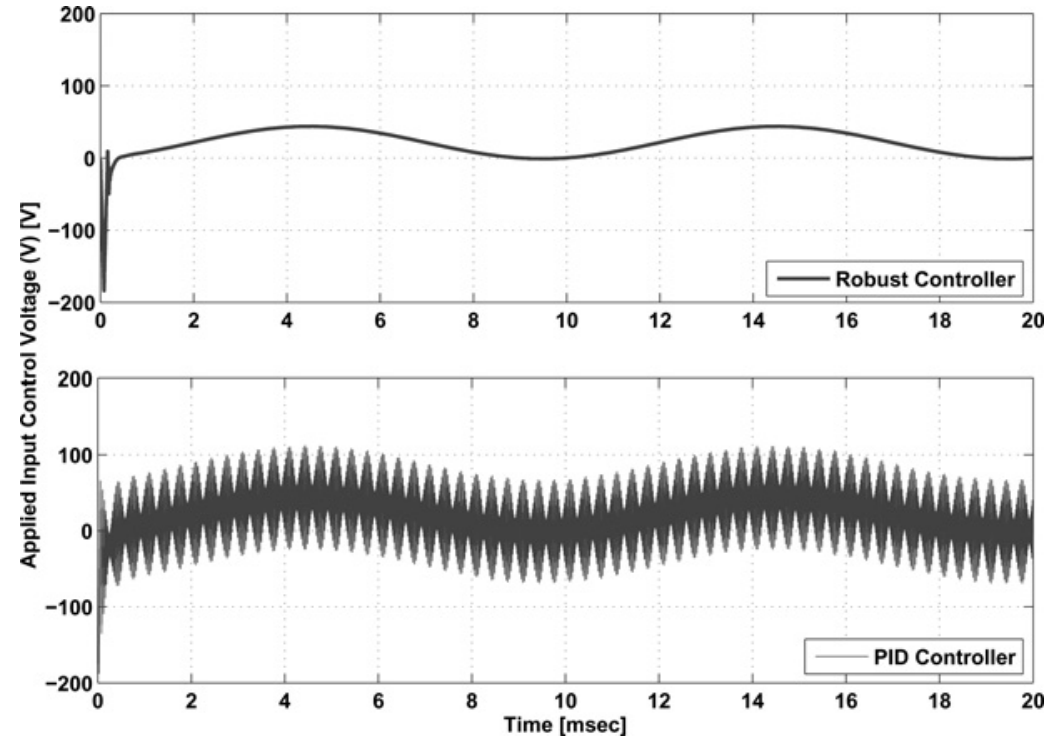

Fig. 11 Voltage $V(t)$ for a desired frequency of $100 \mathrm{~Hz}$ 


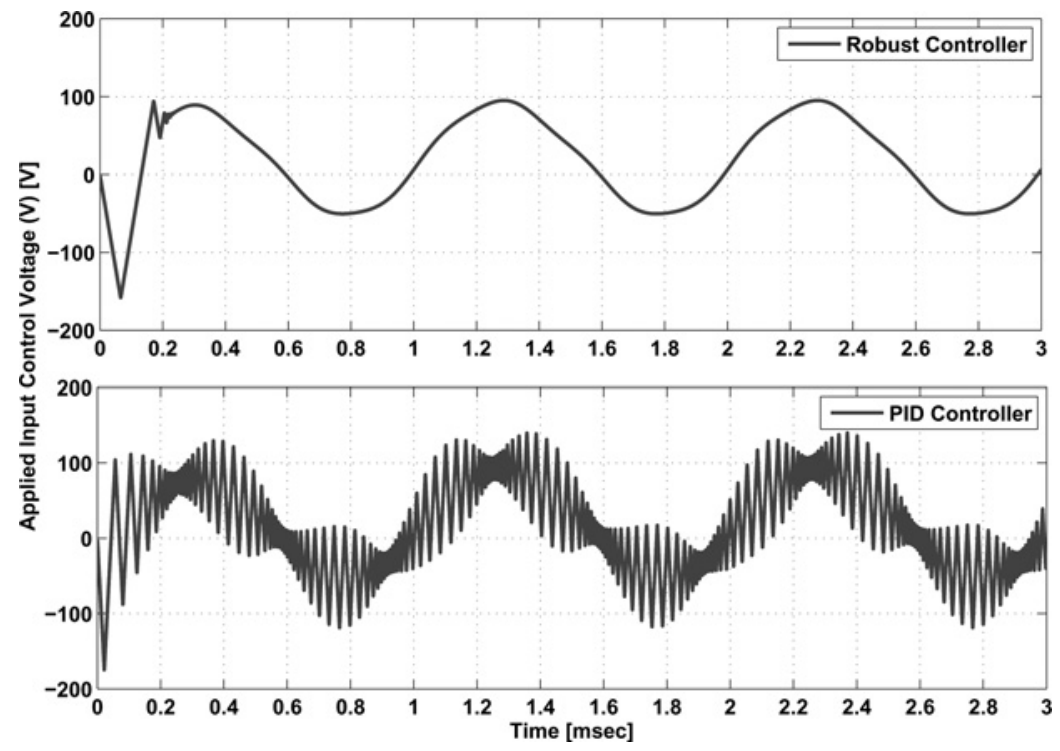

Fig. 12 Voltage $V(t)$ for a desired frequency of $1000 \mathrm{~Hz}$

Table 1 Comparison of tracking error measure for different controllers

\begin{tabular}{cccccccc}
\hline & \multicolumn{3}{c}{ Robust controller } & & \multicolumn{3}{c}{ PID controller } \\
\cline { 2 - 4 } \cline { 6 - 8 } & $10 \mathrm{~Hz}$ & $100 \mathrm{~Hz}$ & $1000 \mathrm{~Hz}$ & & $10 \mathrm{~Hz}$ & $100 \mathrm{~Hz}$ & $1000 \mathrm{~Hz}$ \\
\hline$M_{\mathrm{e}}$ & $7.419 \times 10^{-16}$ & $7.409 \times 10^{-16}$ & $6.623 \times 10^{-16}$ & & $9.713 \times 10^{-16}$ & $9.679 \times 10^{-16}$ & $9.418 \times 10^{-16}$ \\
$M_{\mathrm{v}}$ & 137.9 & 15.89 & 6.71 & & 297.4 & 30.95 & 7.379 \\
\hline
\end{tabular}

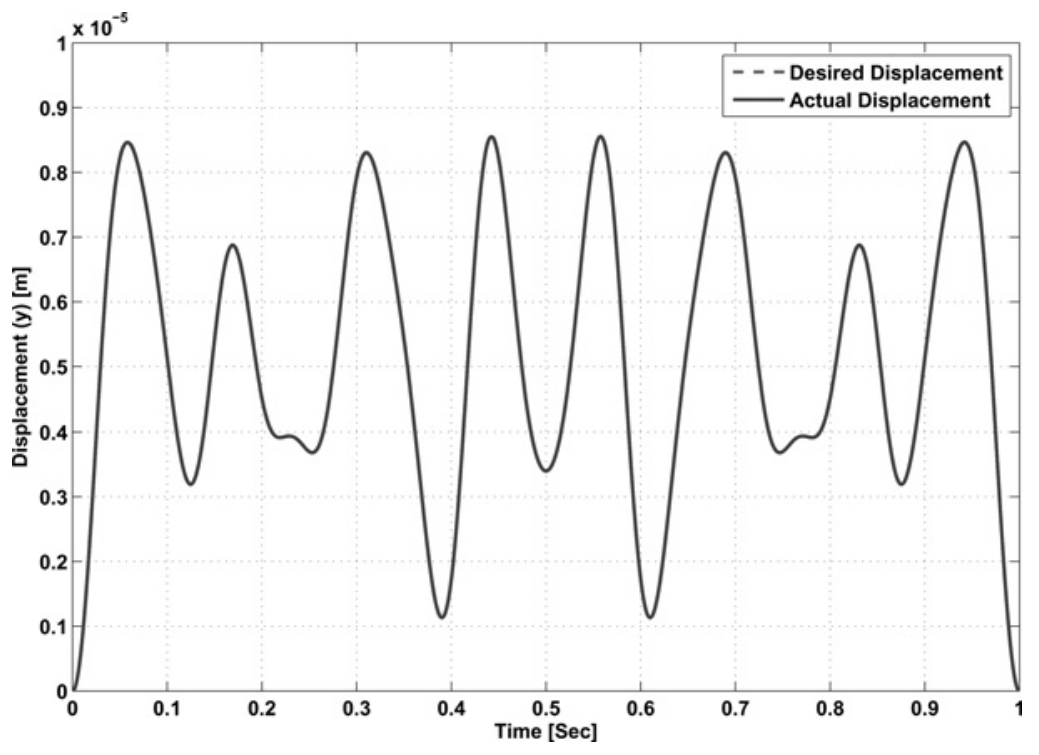

Fig. 13 PZTA multi-frequency desired trajectory $y_{d}(t)$ against actual displacement $y(t)$

Figs. 13-16, respectively. When compared with the results in [43], the proposed robust controller achieved less than $0.66 \%$ tracking error, while the sliding mode controller in
[43] achieved less than 1.9\% (as reported in [43]). This result clearly indicates that the proposed robust controller outperforms similar controllers in the literature. 


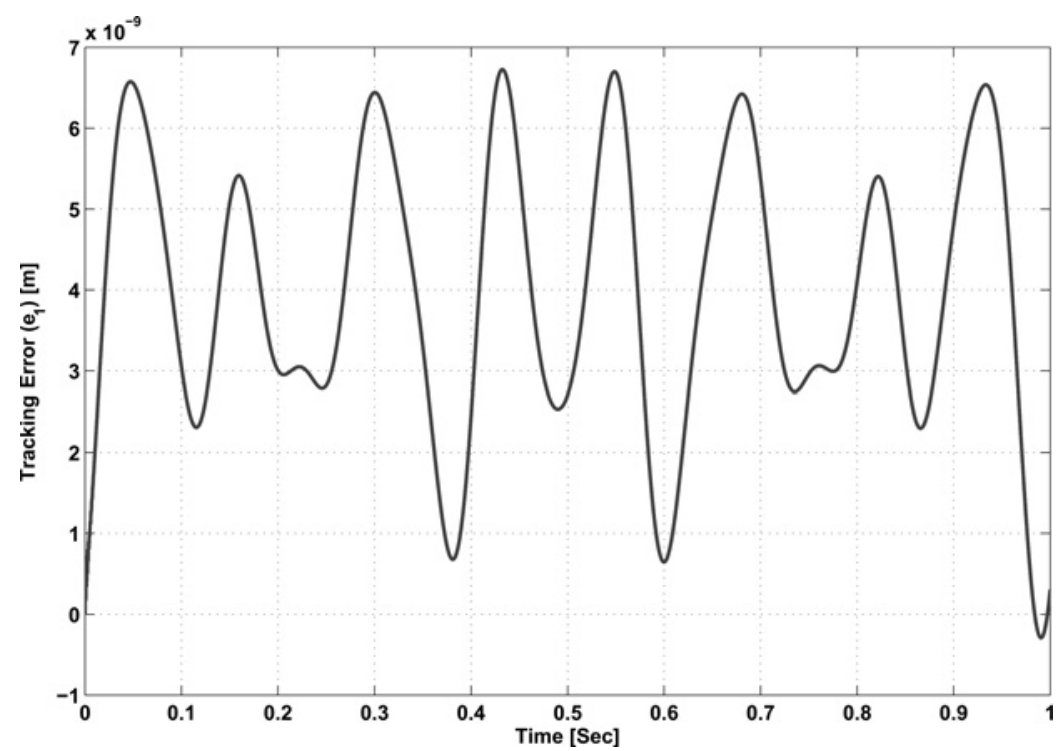

Fig. 14 PZTA tracking error e(t) for the multi-frequency desired trajectory

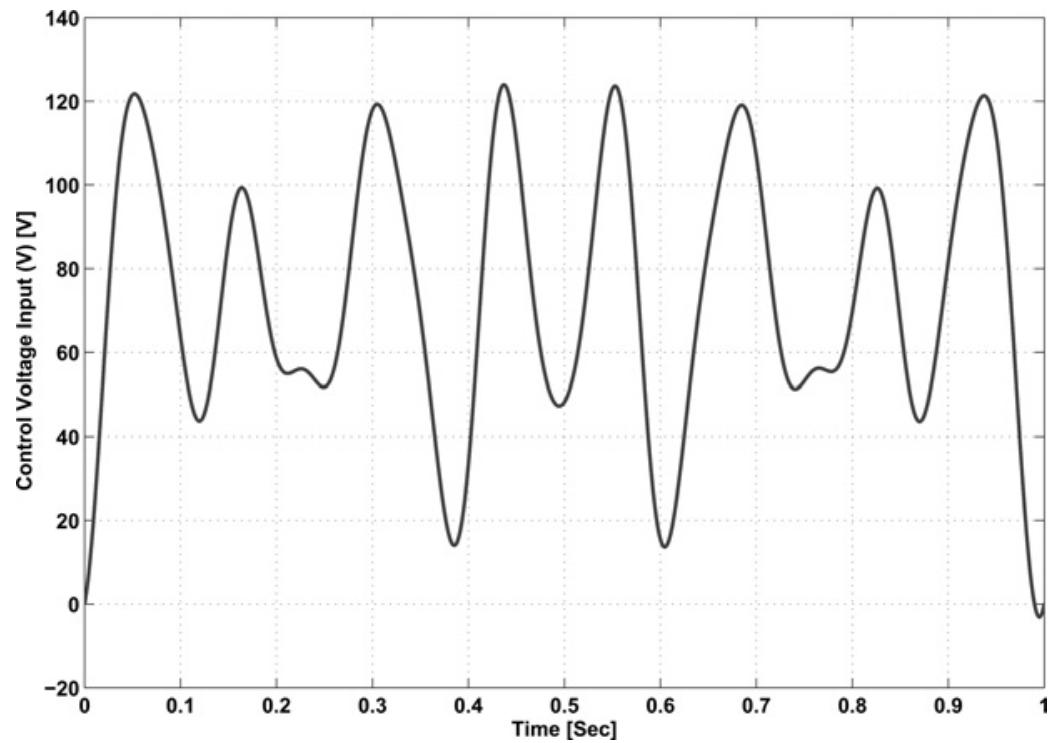

Fig. 15 Voltage $V(t)$ for the multi-frequency desired trajectory

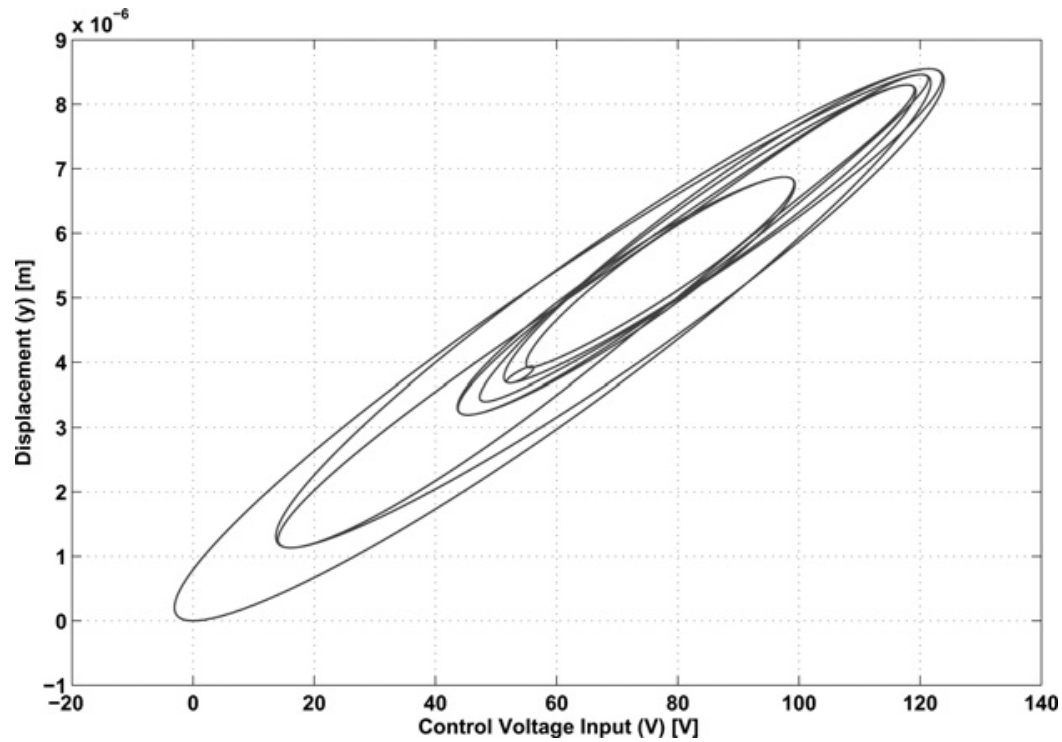

Fig. 16 Hysteresis response for the multi-frequency desired trajectory 


\section{Conclusion}

In this paper, a non-linear robust controller for a PZTA was developed to guarantee that PZTA's effective tip is driven to track a desired trajectory. The PZTA's charge feedback along with the partial knowledge of the hysteresis model was utilised in the design of the non-linear robust control strategy. The Coleman-Hodgdon hysteresis model [45] was utilised in the dynamic model of the PZTA. Lyapunovbased analysis tools were utilised to prove that the tip tracking error is practically regulated to zero. Representative numerical results were presented to illustrate the proof of concept of the proposed robust controller.

\section{Acknowledgments}

A preliminary version of this paper has appeared in the proceedings of the American Control Conference, New York, NY, USA, 2007 [49].

\section{References}

1 Hwang, C., Jan, C.: 'State estimator-based feedback control for a class of piezoelectric systems with hysteresis nonlinearity', IEEE Trans. Syst. Man Cybern. A, 2005, 35, (5), pp. 654-664

2 Liaw, H., Oetomo, D., Shirinzadeh, B., Alici, G.: 'Robust motion tracking control of piezoelectric actuation systems'. Proc. IEEE Int. Conf. on Robotics and Automation, Orlando, FL, USA, 2006, pp. 1414-1419

3 Liu, M., Tong, J., Wang, L., Cui, T.: 'Theoretical analysis of the sensing and actuating effects of piezoelectric multimorph cantilevers', Microsyst. Technol., 2006, 12, pp. 335-342

$4 \mathrm{Xu}$, Y., Meckl, P.: 'Time-optimal motion control of piezoelectric actuator: stm application'. Proc. American Control Conf., Boston, MA, USA, 2004, pp. 4849-4854

5 Daniele, A., Salapaka, S., Salapaka, M., Dahleh, M.: 'Piezoelectric scanners for atomic force microscopes: design of lateral sensors, identification and control'. Proc. American Control Conf., San Diego, CA, USA, 1999

6 Schitter, G., Stemmer, A.: 'Identification and open-loop tracking control of a piezoelectric tube scanner for high speed scanning-probe microscopy', IEEE Trans. Control. Syst. Technol., 2004, 12, (3), pp. 449-454

7 Yee, Y., Nam, H., Lee, S., Bu, J., Jeon, Y., Cho, S.: 'Pzt actuated micromirror for nano-tracking of laser beam for high-density optical data storage'. Proc. 30th Korea-Russia Int. Conf. on MEMS, Miyajaki, Japan, 2000, pp. 435-440

8 Chiang, M., Lee, L., Huang, K.: 'Development of a hydraulicpiezoelectric-actuator for hybrid positioning control with large stroke, high loading and sub-micrometer accuracy'. Proc. IEEE Int. Conf. on Mechatronics, Taipei, Taiwan, 2005, pp. 45-49

9 Abramovitch, D., Franklin, G.: 'A brief history of disk drive control', IEEE Control Syst. Mag., 2002, 22, (3), pp. 28-42

10 Lau, G.K., Du, H.: 'A piezoelectric micro-actuator with extended baseplate for hdd', Microsyst. Technol., 2005, 11, pp. 598-605

11 Suzuki, K., Akimatsu, T., Sasaki, K., Kurita, M., 'Active-head sliders using piezoelectric thin films for flying height control', Microsyst. Technol., 2005, 11, pp. 836-842

12 Mori, S., Furuya, M., Naganawa, A., Shibuya, Y., Obinata, G., Ouchi, $\mathrm{K}$.: 'Nano-motion actuator with large working distance for precise track following', Microsyst. Technol., 2007, 13, pp. 873-881

13 Cho, W.-I., Park, N.-C., Yang, H., Park, Y.-P.: 'Swing-arm-type pzt dual actuator with fast seeking for optical disk drive', Microsyst. Technol., 2002, 8, pp. 139-148

14 Chan, K.W., Liao, W.H.: 'Self-sensing actuators with passive damping for adaptive vibration control of hard disk drives', Microsyst. Technol., 2009, 15, pp. 355-366

15 Mori, S., Hoshino, T., Tada, H., Obinata, G., Ouchi, K.: 'Linear actuator for precise track following’, Microsyst. Technol., 2005, 11, pp. $718-727$

16 Main, J.A., Garcia, E.: 'Piezoelectric stack actuators and control system design: strategies and pitfalls', J. Guid. Control Dyn., 1997, 20, (3), pp. 479-485
17 Vautier, B., Moheimani, S.: 'Charge driven piezoelectric actuators for structural vibrational control: issues and implementation', J. Smart Mater. Struct., 2005, 14, pp. 575-586

18 Huang, Y., Lin, D.: 'Tracking control of a piezoelectric actuator based on experiment hysteretic modeling'. Proc. IEEE/ASME Int Conf. on Advanced Intelligent Mechatronics, Kobe, Japan, 2003, pp. 301-313

19 Devasia, S., Eleftheriou, E., Mohemimani, S.: 'A survey of control issues in nanopositioning', IEEE Trans. Control Syst. Technol., 2007, 15, (5), pp. 802-823

20 Bashash, S., Jalili, N.: 'Robust multiple frequency trajectory tracking control of piezoelectrically driven micro/nanopositioning systems', IEEE Trans. Control Syst. Technol., 2007, 15, (5), pp. 867-878

21 Yeh, T., Lu, S., Wu, T.: 'Modeling and identification of hysteresis in piezoelectric actuators', ASME J. Dyn. Syst. Meas. Control, 2006, 128, pp. 189-196

22 Ji, H., Yang, S., Wu, Z., Yan, G.: 'Precision control of piezoelectric actuator using support vector regression nonlinear model and neural networks'. Proc. Fourth Int. Conf. on Machine Learning and Cybernetics, Guangzhou, China, 2005, pp. 1186-1191

23 Lining, S., Changhai, R., Weibin, R., Liguo, C.: 'Tracking control of piezoelectric actuator based on a new mathematical model', $J$. Micromech. Microeng., 2004, 14, pp. 1439-1444

24 Shieh, H., Lin, F., Huang, P., Teng, L.: 'Adaptive displacement control with hysteresis modeling for piezoactuated positioning mechanism', IEEE Trans. Ind. Electron., 2006, 53, (3), pp. 905-914

25 Ru, C., Chen, L., Shao, B., Rong, W., Sun, L.: 'A hysteresis compensation method of piezoelectric actuator: model, identification and control', Control Eng. Pract., 2009, 17, pp. 1107-1114

26 Kaizuka, H., Sui, B.: 'A simple way to reduce hysteresis and creep when using piezoelectric actuators', Jpn. J. Appl. Phys., 1988, 27, (5), pp. L773-L776

27 Newcomb, C., Flinn, I.: 'Improving the linearity of piezoelectric ceramic actuators', Electron. Lett., 1982, 18, (11), pp. 442-444

28 Furutani, K., Urushibata, M., Mohri, N.: 'Displacement control of piezoelectric element by feedback of induced charge', J. Nanotechnol., 1998, 9, pp. 93-98

29 Main, J., Garcia, E., Newton, D.: 'Precision position control of piezoelectric actuators using charge feedback', J. Guid. Control Dyn., 1995, 18, (5), pp. 1068-1073

30 Ahn, H.: 'Design of a repetitive control system for a piezoelectric actuator based on the inverse hysteresis model'. Proc. Fourth Int. Conf. on Control and Automation, Montreal, Canada, 2003, pp. 128-132

31 Stepanenko, Y., Su, C.: 'Intelligent control of piezoelectric actuators'. Proc. IEEE Int. Conf. on Decision and Control, Tampa, FL, USA, 1998, pp. 4234-4239

32 Wai, R., Lee, J.: 'Intelligent motion control for linear piezoelectric ceramic motor drive', IEEE Trans. Syst. Man Cybern. B, 2004, 34, (5), pp. 2100-2111

33 Lee, B., Yang, S., Ahn, K.: 'Precision control of piezoelectric actuator using inverse hysteresis model and neuro control'. Proc. Seventh Korea-Russia Int. Symp. on Science and Technology, Ulsan, Korea, 2003, pp. 273-238

34 Chen, B., Lee, T., Hang, C., Guo, Y., Weerasooriya, S.: 'An h $\mathrm{\infty}_{\infty}$ almost disturbance decoupling robust controller design for a piezoelectric bimorph actuator with hysteresis', IEEE Trans. Contr. Syst. Technol., 1999, 7, (2), pp. 160-174

35 Choi, G., Kim, H., Choi, G.: 'A study on position control of piezoelectric actuators'. Proc. IEEE Int. Symp. on Industrial Electronics, Guimaraes, Portugal, 1997, pp. 851-855

36 Hwang, C., Chen, Y., Jan, C.: 'Trajectory tracking of largedisplacement piezoelectric actuators using nonlinear observer-based variable structure control', IEEE Trans. Control Syst. Technol., 2005, 13, (1), pp. 56-66

37 Wu, Y., Zou, Q.: 'Iterative control approach to compensate for the hysteresis and the vibrational dynamics effects of piezo actuators' Proc. American Control Conf., Minneapolis, MN, USA, 2006, pp. 424-429

38 Liaw, H., Shirinzadeh, B., Smith, J.: 'Robust motion tracking control of piezo-driven flexure-based four-bar mechanism for micro/nano manipulation', Mechatronics, 2008, 18, pp. 111-120

39 Liaw, H., Shirinzadeh, B., Smith, J.: 'Robust adaptive motion tracking control of piezoelectric actuation systems for micro/nano manipulation'. Proc. IEEE Int. Conf. Robotics and Automation, Roma, Italy, 2007, pp. 1110-1115

40 Tsukagoshi, H., Kitagawa, A., Segawa, M.: 'Robust $h_{\infty}$ control of hystresis in a piezoelectric stack actuator'. Proc. 17th IFAC World Congress, Seoul, Korea, 2008, pp. 1996-2001

41 Liaw, H., Shirinzadeh, B., Smith, J.: 'Sliding-mode enhanced adaptive motion tracking control of piezoelectric actuation systems for 
micro/nano manipulation', IEEE Trans. Control Syst. Technol., 2008, 16, (4), pp. 826-833

42 Wu, Y., Zou, Q.: 'Robust inversion-based 2 dof control design for output tracking: piezoelectric-actuator example', IEEE Trans. Control Syst. Technol., 2009, 17, (5), pp. 1069-1082

43 Bashash, S., Jalili, N.: 'Real-time identification of piezoelectric actuator nonlinearities with application to precision trajectory control'. Proc. American Control Conf., Minneapolis, MN, USA, 2006, pp. 3308-3313

44 Naganawa, A., Mori, S., Tada, H., Shibuya, Y., Obinata, G., Ouchi, K.: 'Finite element method analysis and control of micro actuator with piezoelectric element for spinstand', Microsyst. Technol., 2005, 11, pp. 606-611

45 Coleman, B., Hodgdon, M.: 'On a class of constitutive relations for ferromagnetic hysteresis', Arch. Ration. Mech. Anal., 1987, 99, (4), pp. 375-396

46 Adriaens, H., De-Koning, W., Banning, R.: 'Modeling piezoelectric actuators', IEEE/ASME Trans. Mechatronics, 2000, 5, (4), pp. 331-341

47 Coleman, B., Hodgdon, M.: 'A constitutive relation for rateindependent hysteresis in ferromagnetically soft materials', Int. J. Eng. Sci., 1986, 24, (6), pp. 897-919

48 Xian, B., de Queirioz, M.S., Dawson, D.M., McIntyre M.L.: 'A discontinuous output feedback controller and velocity observer for nonlinear mechanical systems', Automatica, 2004, 40, (4), pp. 695-700

49 Salah, M.H., McIntyre, M.L., Dawson, D.M., Wagner, J.R.: 'Robust tracking control for a piezoelectric actuator'. Proc. American Control Conf., New York, NY, USA, 2007, pp. 106-111

\section{Appendix 1: Proof of Property 6}

Lemma 1: Based on the definitions presented in (3) and (4), the function $d\left(V_{\mathrm{h}}\right)$ can be upper bounded as shown in (5) if $\lim _{V_{\mathrm{h}} \longrightarrow-\infty} d\left(V_{\mathrm{h}}\right)=\lim _{V_{\mathrm{h}} \longrightarrow+\infty} d\left(V_{\mathrm{h}}\right)=0$.

Proof: Based on the status of $V_{\mathrm{h}}(t)$, two cases arise for the hysteresis model as in (3) and (4).

Case I: When $V_{\mathrm{h}}(t)$ is increasing and $V_{\mathrm{h}}(t)>V_{\mathrm{h}_{o}}$, the limit of the right-hand side of the expression in (3) can be written as follows

$$
\begin{aligned}
& \lim _{V_{\mathrm{h}} \longrightarrow+\infty}\left(\left[q_{o}-f\left(V_{\mathrm{h}_{o}}\right)\right] e^{-\delta\left(V_{\mathrm{h}}-V_{\mathrm{h}_{o}}\right)}\right. \\
& \left.+e^{-\delta V_{\mathrm{h}}} \int_{V_{\mathrm{h}_{o}}}^{V_{\mathrm{h}}}\left(g(\tau)-f^{\prime}(\tau)\right) e^{\delta \tau} \mathrm{d} \tau\right)
\end{aligned}
$$

since $q_{o}$ and $f\left(V_{\mathrm{h}_{o}}\right)$ are constants, the expression in (44) can be rewritten as follows

$$
\begin{aligned}
& {\left[q_{o}-f\left(V_{\mathrm{h}_{o}}\right)\right]} \\
& \quad \lim _{\mathrm{h}_{\mathrm{h}} \longrightarrow+\infty} e^{-\delta\left(V_{\mathrm{h}}-V_{\mathrm{h}_{o}}\right)} \\
& \quad+\lim _{V_{\mathrm{h}} \longrightarrow+\infty} e^{-\delta V_{\mathrm{h}}} \lim _{V_{\mathrm{h}} \longrightarrow+\infty} \int_{V_{\mathrm{h}_{o}}}^{V_{\mathrm{h}}}\left(g(\tau)-f^{\prime}(\tau)\right) e^{\delta \tau} \mathrm{d} \tau
\end{aligned}
$$

It is clear that $\lim _{V_{\mathrm{h}} \rightarrow+\infty} e^{-\delta\left(V_{\mathrm{h}}-V_{\mathrm{h}_{o}}\right)}=0$ and $\lim _{V_{\mathrm{h}} \rightarrow+\infty}$ $e^{-\delta V_{\mathrm{h}}}=0$ and from Properties 4 and 5, the following bound can be obtained for the integral term in (45)

$$
\left|\int_{V_{\mathrm{h}_{o}}}^{\infty}\left(g(\tau)-f^{\prime}(\tau)\right) e^{\delta \tau} \mathrm{d} \tau\right| \leq \eta_{1}
$$

where $\eta_{1} \in \mathbb{R}^{+}$is a constant. Hence, it is clear that $\lim _{V_{\mathrm{h}} \longrightarrow+\infty} d\left(V_{\mathrm{h}}\right)=0$.

Case II: When $V_{\mathrm{h}}(t)$ is decreasing and $V_{\mathrm{h}}(t)<V_{\mathrm{h}_{o}}$, the limit of the right-hand side of the expression in (4) can be written as follows

$$
\begin{aligned}
\lim _{V_{\mathrm{h}} \longrightarrow-\infty}\left(\left[q_{o}-f\left(V_{\mathrm{h}_{o}}\right)\right] e^{-\delta\left(V_{\mathrm{h}_{o}}-V_{\mathrm{h}}\right)}\right. & \\
& \left.+e^{\delta V_{\mathrm{h}}} \int_{V_{\mathrm{h}_{o}}}^{V_{\mathrm{h}}}\left(f^{\prime}(\tau)-g(\tau)\right) e^{-\delta \tau} \mathrm{d} \tau\right)
\end{aligned}
$$

By following the same steps in Case I, the limit in (47) can be rewritten as follows

$$
\begin{aligned}
& {\left[q_{o}-f\left(V_{\mathrm{h}_{o}}\right)\right] \lim _{V_{\mathrm{h}} \longrightarrow-\infty} e^{-\delta\left(V_{\mathrm{h}_{o}}-V_{\mathrm{h}}\right)}} \\
& \quad+\lim _{V_{\mathrm{h}} \longrightarrow-\infty} e^{\delta V_{\mathrm{h}}} \lim _{V_{\mathrm{h}} \longrightarrow-\infty} \int_{V_{\mathrm{h}_{o}}}^{V_{\mathrm{h}}}\left(f^{\prime}(\tau)-g(\tau)\right) e^{-\delta \tau} \mathrm{d} \tau
\end{aligned}
$$

It is clear that $\lim _{V_{\mathrm{h}} \longrightarrow-\infty} e^{-\delta\left(V_{\mathrm{h}_{o}}-V_{\mathrm{h}}\right)}=0$ and $\lim _{V_{\mathrm{h}} \longrightarrow-\infty}$ $e^{\delta V_{\mathrm{h}}}=0$ and from Properties 4 and 5, the upper bound in (46) is satisfied for Case II too. Hence, it is clear that $\lim _{V_{\mathrm{h}} \longrightarrow-\infty} d\left(V_{\mathrm{h}}\right)=0$. From the results obtained in Cases I and II, it is obvious that $\left|d\left(V_{\mathrm{h}}\right)\right| \leq \eta_{\mathrm{d}}$.

\section{Appendix 2: Proof of Theorem 1}

Let $P(z, t) \in \mathbb{R}$ denote the following non-negative function

$$
P \triangleq \frac{1}{2} e^{2}+\frac{1}{2} m r^{2}
$$

Note that the right-hand side of (49) can be bounded as follows

$$
\lambda_{1}\|z\|^{2} \leq P(z, t) \leq \lambda_{2}\|z\|^{2}
$$

where $\lambda_{1}, \lambda_{2} \in \mathbb{R}^{+}$are bounding constants. After taking the time derivative of (49), the following expression can be written as

$$
\begin{aligned}
\dot{P}= & -\alpha e^{2}-k_{r} r^{2}+\left(\frac{T_{\mathrm{em}}}{C_{\mathrm{c}}}\right) r s+r N_{\mathrm{d}}+r \tilde{N} \\
& -\frac{1}{\varepsilon} \rho(\|z\|)^{2}\|z\|^{2} r^{2}
\end{aligned}
$$

where (12) and (33) were utilised. By utilising (30), and the triangle inequality, $\dot{P}(t)$ can be upper bounded as follows

$$
\begin{aligned}
\dot{P} \leq & -\alpha|e|^{2}-k_{r}|r|^{2}+\left(\frac{T_{\mathrm{em}}}{C_{\mathrm{c}}}\right)|r||s|+|r|\left|N_{\mathrm{d}}\right| \\
& +|r| \rho(\|z\|)\|z\|-\frac{1}{\varepsilon} \rho(\|z\|)^{2}\|z\|^{2}|r|^{2}
\end{aligned}
$$

Note that the terms $|r(\cdot)||s(\cdot)|$ and $|r(\cdot)|\left|N_{\mathrm{d}}(\cdot)\right|$ can be upper bounded as follows

$$
\begin{aligned}
|r||s| & \leq \frac{1}{\delta 1}|r|^{2}+\delta_{1}|s|^{2} \\
|r|\left|N_{\mathrm{d}}\right| & \leq \frac{1}{\delta_{2}}|r|^{2}+\delta_{2}\left|N_{\mathrm{d}}\right|^{2}
\end{aligned}
$$

where $\delta_{1}, \delta_{2} \in \mathbb{R}^{+}$are constants. After utilising (53) and (54), the expression on the right-hand side of (52) can be upper bounded as follows

$$
\dot{P} \leq-\lambda_{3}\|z\|^{2}+\rho(\|z\|)\|z\||r|\left(1-\frac{1}{\varepsilon} \rho(\|z\|)\|z\||r|\right)+\varepsilon_{o}
$$

where $\lambda_{3} \triangleq \min \left\{\alpha,\left(k_{r}-\left(T_{\mathrm{em}} / C_{\mathrm{c}} \delta_{1}\right)-\left(1 / \delta_{2}\right)\right)\right\}$, provided $k_{r}$ is selected such that $k_{r} \geq\left(\left(T_{\mathrm{em}} / C_{\mathrm{c}} \delta_{1}\right)+\left(1 / \delta_{2}\right)\right)$. The 
variable $\varepsilon_{o} \in \mathbb{R}^{+}$is defined as follows

$$
\varepsilon_{o} \triangleq\left(\frac{T_{\mathrm{em}} \delta_{1}}{C_{\mathrm{c}}}\right)|s|^{2}+\delta_{2}\left|N_{\mathrm{d}}\right|^{2}
$$

The expression in (56) can be upper bounded by the following

$$
\varepsilon_{o} \leq\left(\frac{T_{\mathrm{em}} \delta_{1}}{C_{\mathrm{c}}}\right) \eta_{s}^{2}+\delta_{2} \eta_{N_{\mathrm{d}}}^{2}
$$

where the following bound was utilised

$$
\left|N_{\mathrm{d}}\right| \leq \eta_{N_{\mathrm{d}}}
$$

where $\eta_{s}, \eta_{N_{\mathrm{d}}} \in \mathbb{R}^{+}$are constants, and (23) and Property 6 were utilised. From (55), two cases arise.

Case I: When $(\varepsilon<\rho(\|z\|)\|z\||r|)$, the expression in (55) can be written as follows

$$
\dot{P} \leq-\lambda_{3}\|z\|^{2}+\varepsilon_{o}
$$

where the term $\rho(\|z\|)\|z\||r|(1-1 / \varepsilon \rho(\|z\|)\|z\||r|)$ in (55) will always be negative.

Case II: When $(\varepsilon>\rho(\|z\|)\|z\||r|)$, the expression in (55) can be written as follows

$$
\dot{P} \leq-\lambda_{3}\|z\|^{2}+\varepsilon_{1}
$$

where $\varepsilon_{1} \in \mathbb{R}^{+}$is defined as $\varepsilon_{1} \triangleq \varepsilon+\varepsilon_{o}$.

In both cases, (59) and (60) can be written as follows

$$
\dot{P} \leq-\frac{\lambda_{3}}{\lambda_{1}} P+\varepsilon_{1}
$$

From (61) it is clear that $\|z\|$ is upper bounded as follows

$$
\|z\| \leq \sqrt{\beta_{o} \exp \left(-\beta_{1}\left(t-t_{0}\right)\right)+\beta_{2}\left(1-\exp \left(-\beta_{1}\left(t-t_{0}\right)\right)\right)}
$$

where $\beta_{o}=\lambda_{2} / \lambda_{1}\left\|z\left(t_{o}\right)\right\|^{2}, \quad \beta_{1}=\lambda_{3} / \lambda_{1}$, and $\beta_{2}=\varepsilon_{1} / \lambda_{3}$. From (62) it is clear that $z(t)$ is practically regulated to 0 as $t \longrightarrow \infty$, hence, from $(31), e(t)$ is practically regulated to 0 as $t \longrightarrow \infty$, thus meeting the tracking control objective. It is clear from (50) and (61), it is clear that $P(z, t) \in \mathcal{L}_{\infty}$; hence $z(t), r(t), e(t) \in \mathcal{L}_{\infty}$. From (12), it is clear that $\dot{e}(t) \in \mathcal{L}_{\infty}$. Assumption 4 can be utilised along with (13) and its first time derivative to show that $y(t), \dot{y}(t) \in \mathcal{L}_{\infty}$. Since $z(t)$, $r(t) \in \mathcal{L}_{\infty}$, from (32), it is clear that $q_{\mathrm{d}}(t) \in \mathcal{L}_{\infty}$. From the bounding statements in (19) and (23), it is clear that $q(t) \in$ $\mathcal{L}_{\infty}$, thus $V_{\mathrm{m}}(t) \in \mathcal{L}_{\infty}$. From (21), it is clear that $\bar{V}_{\mathrm{h}}(t) \in \mathcal{L}_{\infty}$. From (6) and (8), it is clear that $q_{p}(t), V_{\mathrm{c}}(t) \in \mathcal{L}_{\infty}$; thus, from (16), it is clear that $V(t) \in \mathcal{L}_{\infty}$. After utilising the above boundedness statements along with (1) and (7), it is clear that $\ddot{y}(t) \in \mathcal{L}_{\infty}$. Standard signal chasing arguments can be used to prove that all remaining closed-loop signals are bounded.

\section{Appendix 3: Upper bound development}

In this appendix, the bound expression in (30) will be proven. The definition in (28) can be rewritten as follows

$$
N\left(y, \dot{y}, e, r, \ddot{y}_{\mathrm{d}}\right)=m \ddot{y}_{\mathrm{d}}+\bar{F}_{L}+\left(1-\alpha^{2} m\right) e+\alpha m r
$$

where (12) was utilised. To further facilitate the subsequent analysis, $N\left(y, \dot{y}_{\mathrm{d}}, 0,0, \ddot{y}_{\mathrm{d}}\right), N\left(y, \dot{y}, 0,0, \ddot{y}_{\mathrm{d}}\right)$ and $N\left(y, \dot{y}, e, 0, \ddot{y}_{\mathrm{d}}\right)$ are added and subtracted to the right-hand side of (27) as follows

$$
\begin{aligned}
\tilde{N}= & {\left[N\left(y, \dot{y}_{\mathrm{d}}, 0,0, \ddot{y}_{\mathrm{d}}\right)-N_{\mathrm{d}}\left(y_{\mathrm{d}}, \dot{y}_{\mathrm{d}}, 0,0, \ddot{y}_{\mathrm{d}}\right)\right] } \\
& +\left[N\left(y, \dot{y}, 0,0, \ddot{y}_{\mathrm{d}}\right)-N\left(y, \dot{y}_{\mathrm{d}}, 0,0, \ddot{y}_{\mathrm{d}}\right)\right] \\
& +\left[N\left(y, \dot{y}, e, 0, \ddot{y}_{\mathrm{d}}\right)-N\left(y, \dot{y}, 0,0, \ddot{y}_{\mathrm{d}}\right)\right] \\
& +\left[N\left(y, \dot{y}, e, r, \ddot{y}_{\mathrm{d}}\right)-N\left(y, \dot{y}, e, 0, \ddot{y}_{\mathrm{d}}\right)\right]
\end{aligned}
$$

After applying the mean value theorem to each bracketed term of (64), the following expression can be obtained

$$
\begin{aligned}
\tilde{N}= & \left.\frac{\partial N\left(\sigma_{1}, \dot{y}_{\mathrm{d}}, 0,0, \ddot{y}_{\mathrm{d}}\right)}{\partial \sigma_{1}}\right|_{\sigma_{1}=v_{1}}\left(y-y_{\mathrm{d}}\right) \\
& +\left.\frac{\partial N\left(y, \sigma_{2}, 0,0, \ddot{y}_{\mathrm{d}}\right)}{\partial \sigma_{2}}\right|_{\sigma_{2}=v_{2}}\left(\dot{y}-\dot{y}_{\mathrm{d}}\right) \\
& +\left.\frac{\partial N\left(y, \dot{y}, \sigma_{3}, 0, \ddot{y}_{\mathrm{d}}\right)}{\partial \sigma_{3}}\right|_{\sigma_{3}=v_{3}}(e-0) \\
& +\left.\frac{\partial N\left(y, \dot{y}, e, \sigma_{4}, \ddot{y}_{\mathrm{d}}\right)}{\partial \sigma_{4}}\right|_{\sigma_{4}=v_{4}}(r-0)
\end{aligned}
$$

where $v_{1} \in\left(y_{\mathrm{d}}, y\right), v_{2} \in\left(\dot{y}_{\mathrm{d}}, \dot{y}\right), v_{3} \in(e, 0)$ and $v_{4} \in(r, 0)$. The right-hand side of (65) can be upper bounded as follows

$$
\begin{aligned}
\tilde{N} \leq & \left|\frac{\partial N\left(\sigma_{1}, \dot{y}_{\mathrm{d}}, 0,0, \ddot{y}_{\mathrm{d}}\right)}{\partial \sigma_{1}}\right|_{\sigma_{1}=v_{1}}|| e \mid \\
& +\left|\frac{\partial N\left(y, \sigma_{2}, 0,0, \ddot{y}_{\mathrm{d}}\right)}{\partial \sigma_{2}}\right|_{\sigma_{2}=v_{2}}|\dot{e}| \\
& +\left|\frac{\partial N\left(y, \dot{y}, \sigma_{3}, 0, \ddot{y}_{\mathrm{d}}\right)}{\partial \sigma_{3}}\right|_{\sigma_{3}=v_{3}}|e| \\
& +\left|\frac{\partial N\left(y, \dot{y}, e, \sigma_{4}, \ddot{y}_{\mathrm{d}}\right)}{\partial \sigma_{4}}\right|_{\sigma_{4}=v_{4}}|| r \mid
\end{aligned}
$$

The partial derivatives in (66) can be calculated from (63) as

$$
\begin{aligned}
& \frac{\partial N\left(\sigma_{1}, \dot{y}_{\mathrm{d}}, 0,0, \ddot{y}_{\mathrm{d}}\right)}{\partial \sigma_{1}}=f_{L_{1}} \\
& \frac{\partial N\left(y, \sigma_{2}, 0,0, \ddot{y}_{\mathrm{d}}\right)}{\partial \sigma_{2}}=f_{L_{2}} \\
& \frac{\partial N\left(y, \dot{y}, \sigma_{3}, 0, \ddot{y}_{\mathrm{d}}\right)}{\partial \sigma_{3}}=1-\alpha^{2} m \\
& \frac{\partial N\left(y, \dot{y}, e, \sigma_{4}, \ddot{y}_{\mathrm{d}}\right)}{\partial \sigma_{4}}=\alpha m
\end{aligned}
$$

where (10) was utilised and the functions $f_{L_{1}}\left(\sigma_{1}, \dot{y}_{\mathrm{d}}\right) \in \mathbb{R}$ and $f_{L_{2}}\left(y, \sigma_{2}\right) \in \mathbb{R}$ are defined as follows

$$
\begin{aligned}
& f_{L_{1}} \triangleq \frac{\partial F_{L}\left(\sigma_{1}, \dot{y}_{\mathrm{d}}\right)}{\partial \sigma_{1}}+\frac{T_{\mathrm{em}}^{2}}{C_{\mathrm{c}}} \\
& f_{L_{2}} \triangleq \frac{\partial F_{L}\left(y, \sigma_{2}\right)}{\partial \sigma_{2}}
\end{aligned}
$$

By noting that

$$
\begin{aligned}
& v_{1}=y-c_{1}\left(y-y_{\mathrm{d}}\right), \quad v_{2}=\dot{y}-c_{2}\left(\dot{y}-\dot{y}_{\mathrm{d}}\right) \\
& v_{3}=e-c_{3}(e-0), \quad v_{4}=r-c_{4}(r-0)
\end{aligned}
$$




\section{www.ietdl.org}

where $c_{i} \in(0,1) \forall i=1,2, \ldots, 4$. From Assumptions 1, 3 and 4 , it is clear that an upper bound for the right-hand side of (67)-(70) can be written as follows:

$$
\begin{aligned}
\left|\frac{\partial N\left(\sigma_{1}, \dot{y}_{\mathrm{d}}, 0,0, \ddot{y}_{\mathrm{d}}\right)}{\partial \sigma_{1}}\right|_{\sigma_{1}=v_{1}} \mid & \leq \rho_{1}(|e|,|r|) \\
\left|\frac{\partial N\left(y, \sigma_{2}, 0,0, \ddot{y}_{\mathrm{d}}\right)}{\partial \sigma_{2}}\right|_{\sigma_{2}=v_{2}} \mid & \leq \rho_{2}(|e|,|r|) \\
\left|\frac{\partial N\left(y, \dot{y}, \sigma_{3}, 0, \ddot{y}_{\mathrm{d}}\right)}{\partial \sigma_{3}}\right|_{\sigma_{3}=v_{3}} \mid & \leq \rho_{3}
\end{aligned}
$$

$$
\left|\frac{\partial N\left(y, \dot{y}, e, \sigma_{4}, \ddot{y}_{\mathrm{d}}\right)}{\partial \sigma_{4}}\right|_{\sigma_{4}=v_{4}} \mid \leq \rho_{4}
$$

where $\rho_{1}(\cdot)$ and $\rho_{2}(\cdot)$ are positive, non-decreasing functions of their arguments and $\rho_{3}$ and $\rho_{4}$ are positive constants. After substituting (73)-(76) into (66), an upper bound for $|\tilde{N}(\cdot)|$ can be expressed as

$$
|\tilde{N}(\cdot)| \leq \rho_{1}(\|z\|)|e|+\rho_{2}(\|z\|)|\dot{e}|+\rho_{3}|e|+\rho_{4}|r|
$$

The expressions in (12) and (31) can now be used to upper bound the right-hand side of (77) as in (30). 
Copyright of IET Control Theory \& Applications is the property of Institution of Engineering \& Technology and its content may not be copied or emailed to multiple sites or posted to a listserv without the copyright holder's express written permission. However, users may print, download, or email articles for individual use. 